\title{
Advanced Asymptomatic Carotid Disease and Cognitive Impairment: An Understated Link?
}

\author{
Irena Martinić-Popović, Arijana Lovrenčić-Huzjan, and Vida Demarin \\ University Department of Neurology, Sestre Milosrdnice University Hospital Center, 10000 Zagreb, Croatia \\ Correspondence should be addressed to Irena Martinić-Popović, irene@mail.inet.hr
}

Received 2 August 2011; Accepted 22 December 2011

Academic Editor: Michael J. Katsnelson

Copyright (C 2012 Irena Martinić-Popović et al. This is an open access article distributed under the Creative Commons Attribution License, which permits unrestricted use, distribution, and reproduction in any medium, provided the original work is properly cited.

\begin{abstract}
Advanced carotid disease is known to be associated with symptomatic cerebrovascular diseases, such as stroke or transient ischemic attack (TIA), as well as with poststroke cognitive impairment. However, cognitive decline often occurs in patients with advanced carotid stenosis without clinically evident stroke or TIA, so it is also suspected to be an independent risk factor for dementia. Neurosonological methods enable simple and noninvasive assessment of carotid stenosis in patients at risk of advanced atherosclerosis. Cognitive status in patients diagnosed with advanced carotid stenosis is routinely not taken into consideration, although if cognitive impairment is present, such patients should probably be called symptomatic. In this paper, we discuss results of some most important studies that investigated cognitive status of patients with asymptomatic advanced carotid disease and possible mechanisms involved in the causal relationship between asymptomatic advanced carotid disease and cognitive decline.
\end{abstract}

\section{Introduction}

While detrimental effects of stroke on cognitive functions have been well documented in the literature, the mechanisms linking advanced carotid disease and impaired cognitive status in patients without symptomatic cerebrovascular incidents are less clear.

Advanced carotid disease is associated with the presence of multiple vascular risk factors, which most often include arterial hypertension, dyslipidemia, cigarette smoking, diabetes, and older age [1-3]. The same risk factors were shown to be associated not only with vascular dementia, but also with neurodegenerative dementia, importantly with Alzheimer's disease [4]. The vascular hypothesis of Alzheimer's disease provides substantial evidence that vascular risk factors play a critical role in the development of cognitive impairment and clinically evident dementia during aging [4]. Vascular dementia and Alzheimer's disease in their pure forms are two ends of a pathologic continuum [5]. However, many studies during the last decade implicated the overlap of their pathologies. It was shown that a substantial proportion of brains who meet neuropathological criteria for Alzheimer's disease also demonstrate lesions typical for vascular pathology, such as cerebral amyloid angiopathy, microvascular degeneration, and periventricular white matter lesions [6, 7]. Results of the seminal "Nun Study" that followed 102 elderly nuns until postmortem showed higher prevalence of clinically expressed dementia in those who met neuropathological criteria for Alzheimer's disease and simultaneously had brain infarcts [8]. Furthermore, in a 3-year follow-up study of stroke patients who were not demented before the stroke, one-third of the patients who developed poststroke dementia were diagnosed as suffering from Alzheimer's disease [9]. There is now substantial and growing evidence from studies of epidemiology, pharmacology, neuroimaging, clinical medicine, microscopic anatomy, and cellular-molecular biology to hypothesize that sporadic Alzheimer's disease is induced or accelerated by impaired cerebral perfusion [10-14].

There are few proposed mechanisms aiming to explain the link between carotid stenosis and cognitive impairment. Carotid stenosis itself could directly cause decreased cognitive functioning or it may act as a marker of cerebral atherosclerosis due to the presence of conventional vascular risk factors in such patients [15]. Preexistent silent cerebral ischemia, lacunar infarcts, or preclinical Alzheimer's disease 
in patients with carotid stenosis may also influence their neuropsychological performance.

According to population studies, carotid disease of various degree can be found in more than $75 \%$ of men and in more than $62 \%$ of women older than 65 years of age. Total prevalence of carotid disease over $50 \%$ in the same age group is $7 \%$ in men and 5\% in women [16]. Repercussions of carotid stenosis/occlusion on intracranial hemodynamics have been recognised by Spencer and Reid [17]. According to their study and the known "Spencer curve," in mild and moderate carotid stenosis brain blood perfusion remains stable, until high-grade carotid stenosis occurs [18]. Besides cerebral microembolisation, proposed mechanisms of cognitive impairment in individuals with advanced carotid disease thus include chronic hypoperfusion.

During normal aging process, cerebral blood flow modestly diminishes and can decrease up to $20 \%$ by the age of $65[19,20]$. Aging is often accompanied by increase in minor memory problems which are followed by more pronounced cognitive impairment $[21,22]$. Since cognitive decline during aging is commonly prodromal to Alzheimer's disease or to vascular dementia ( VaD), treating disease harbingers, such as cerebrovascular and cardiovascular risk factors, may be one of the most important prevention strategies aimed at either dementia [14]. Mild, diffuse brain damage related to chronic hypoperfusion may affect the brain's ability to process information quickly and efficiently even in the absence of discrete white matter lesions [23]. Not surprisingly, carotid stenosis in asymptomatic individuals can produce a reduction of subtle cognitive abilities involving the function of the hemisphere ipsilateral to the carotid stenosis [4].

Despite the possible poor cognitive outcome, in the absence of stroke or TIA, patients diagnosed with advanced carotid disease are considered asymptomatic and their cognitive status is not routinely assessed. Decreased cognitive functioning in those patients so far has not been recognised as possible factor that could influence therapeutic decision related to the carotid disease.

\section{Assessment of Cognitive Functioning in Patients with Carotid Stenosis}

A number of previous studies suggested that in stroke/TIAfree patients severe carotid disease might be associated with subtle cognitive changes, but results of studies have not been consistent [3, 16, 24-26]. The principal reasons for somewhat conflicting results are differences in study designs, mostly small sample size, and nonconsistent selection of cognitive tests performed. Only few studies of cognitive status, mostly on a small number of patients, were conducted in patients with advanced carotid disease $[3,16,25]$. Patients with carotid disease were shown to have significantly worse scores on cognitive tests than matched controls. Cognitive problems experienced by patients with advanced carotid disease are mostly subtle and not severe enough to interfere with their daily activities, therefore remaining unrecognized by their closest family or friends [15]. Such changes are insufficient to meet criteria for dementia and therefore correspond to mild cognitive impairment (MCI).
Although modern cognitive neuropsychology has developed sophisticated tests in order to assess the different cognitive functions, most studies of cognitive impairment in patients with carotid disease used simple Mini-Mental-State Examination (MMSE) as a routine cognitive measure [27]. Others used more extensive neuropsychological instruments in order to analyze cognitive domains $[16,24]$.

The Mini-Mental State Examination (MMSE) remains the most commonly applied screening test in clinical practice. It includes five cognitive domains (with a maximum score of 30 points); however it does not contain much capacity to test frontal/executive or visuospatial functions. Another problem with MMSE is its low sensitivity in detecting subtle, early-phase cognitive changes so it is not recommended for screening for $\mathrm{MCI}[28,29]$. Patients with discrete decline of cognitive status (such as patients with carotid disease) often find tasks involved in MMSE insultingly simple.

Neuropsychological testing with standardized cognitive tests is time consuming and difficult to implement in busy clinical settings; therefore some more recent studies of cognitive functioning in patients with carotid disease successfully used Montreal Cognitive Assessment (MoCA) as a simple and brief screening tool with high sensitivity for subtle cognitive impairment [29-31]. The MoCA is a 30 -point test involving eight cognitive domains in which results below cut-off score indicate cognitive impairment. A workshop group of NIH and Canadian Stroke Consortium proposed the selected MoCA subtests, including a 5-word immediate and delayed memory test, a 6-item orientation task, and a 1-letter phonemic fluency test (the letter F), for a routine use in a five-minute protocol aiming to assess individuals with possible vascular cognitive impairment [32].

Due to the lack of inclusion of frontal lobe tests in commonly applied MMSE, it appears that MoCA could be recommended as more appropriate tool for the assessment of cognitive changes in patients with carotid stenosis. Although the aim of routinely used screening tests is to detect the presence of cognitive impairment and not to distinguish between its causes, patients with predominantly vascular etiology of subtle cognitive impairment and probable significant frontal lobe pathology could be tested more accurately using MoCA as it contains both subtests with executive function and with memory.

Despite the growing number of studies that have investigated cognitive impairment in patients with carotid disease, correct pathophysiological interpretation of the underlying mechanisms remains unclear. The relationship of decreased cognitive status and asymptomatic carotid stenosis was published from the observations from the Tromsø study, including results from a heterogeneous group of patients with bilateral carotid stenosis of $\geq 35 \%$ [3]. Their performances in a battery of extensive neuropsychological tests were compared with results from subjects without carotid stenoses. In this study, patients with carotid disease achieved significantly lower levels of performance in several subsets of cognitive tests. In a large number of 4006 asymptomatic patients participating in the Cardiovascular Health Study 
and diagnosed with various degrees of carotid stenosis, Johnston et al. used the modified MMSE in order to evaluate the cognitive status [16]. This study found that advanced stenosis $(\geq 75 \%)$ of the left internal carotid artery was associated with a cognitive impairment and cognitive decline during the followup, but no significant correlation was detected for right-sided stenoses. As the correlation of a left carotid stenosis and reduced cognitive functioning was observed even in participants without evidence of cerebral infarction on MRI, it is likely that an asymptomatic carotid stenosis may be an independent risk factor for a cognitive impairment. In our pilot study, as well as in a study performed on a larger number of 70 patients diagnosed with advanced carotid stenosis or occlusion, we have shown cognitive impairment detectable by MoCA, but not by MMSE [30, 31]. MoCA subtests analysis in patients with carotid disease demonstrated significantly lower subscores in multiple domains, including visuospatial and executive functions, attention, and delayed recall [31]. Similar results were achieved in the Troms $\varnothing$ study where patients with asymptomatic carotid stenosis scored worse at attention, memory, psychomotor speed, and motor functioning [3]. However, comparing of studies results presents a major problem due to differences in cognitive testing methods. Silvestrini et al. reported on reduced cognitive scores at verbal fluency in patients with severe left-sided ICA stenosis while those with right-sided stenosis performed worse at abstract reasoning and executive functions [33]. This is similar to the results from Bossema et al. [34]. According to Komulainen et al. the degree of carotid artery stenosis correlates with the extent of cognitive loss [35]. In the Framingham Offspring study, in a followup of subgroup of 1971 participants with carotid disease, carotid disease was found to be associated with significant poorer performance on cognitive tests in asymptomatic subjects without signs of silent cerebral infarcts or white matter hyperintensities on brain MRI [36]. Study that included Canadian First Nations population who have a larger proportions of vascular risk factors showed contradictory results as individuals with left-sided carotid disease were less likely to have cognitive impairment. However, cognitive measurements were performed using only the "Trial Making Test" and only the small proportion of patients included were diagnosed with advanced carotid disease [37].

\section{Conclusions}

The effect of carotid stenosis on cognitive functioning remains far from being unambiguously explained. Systematic and carefully designed prospective studies with defined and consecutive inclusion of patients screened for concomitant disorders that might influence cognitive functioning, the use of uniformed neuropsychological testing, and appropriate neuroimaging (MRI) assessment in patients with carotid disease are still lacking. However, despite differences in studies results, we believe that asymptomatic advanced carotid stenosis should be considered an independent risk factor for the decline in cognitive functioning. If asymptomatic advanced carotid stenosis causes cognitive impairment, the threshold for operative treatment may change and reduction in risk for cognitive impairment may be an important benefit of interventions to treat carotid disease [15]. For the time being, effective treatment of vascular risk factors remains the most important clinical recommendation for stroke/TIAfree patients with carotid stenosis. As cognitive problems in stroke/TIA-free patients with carotid disease are so far often underestimated in routine clinical practice, the use of MoCA could aid in better recognition of this problem.

\section{References}

[1] D. H. O'Leary, J. F. Polak, R. A. Kronmal et al., "Distribution and correlates of sonographically detected carotid artery disease in the Cardiovascular Health Study," Stroke, vol. 23, no. 12, pp. 1752-1760, 1992.

[2] J. S. Fine-Edelstein, P. A. Wolf, D. H. O’Leary et al., "Precursors of extracranial carotid atherosclerosis in the Framingham Study," Neurology, vol. 44, no. 6, pp. 1046-1050, 1994.

[3] E. B. Mathiesen, K. Waterloo, O. Joakimsen, S. J. Bakke, E. A. Jacobsen, and K. H. Bønaa, "Reduced neuropsychological test performance in asymptomatic carotid stenosis: the Tromsø Study," Neurology, vol. 62, no. 5, pp. 695-701, 2004.

[4] J. C. de la Torre, "Vascular risk factor detection and control may prevent Alzheimer's disease," Ageing Research Reviews, vol. 9, no. 3, pp. 218-225, 2010.

[5] R. N. Kalaria and C. Ballard, "Overlap between pathology of Alzheimer disease and vascular dementia," Alzheimer Disease and Associated Disorders, vol. 13, no. 3, supplement, pp. S115S123, 1999.

[6] R. Kalaria, "Similarities between Alzheimer's disease and vascular dementia," Journal of the Neurological Sciences, vol. 203-204, pp. 29-34, 2002.

[7] R. N. Kalaria, "The role of cerebral ischemia in Alzheimer's disease," Neurobiology of Aging, vol. 21, no. 2, pp. 321-330, 2000.

[8] D. A. Snowdon, L. H. Greiner, J. A. Mortimer, K. P. Riley, P. A. Greiner, and W. R. Markesbery, "Brain infarction and the clinical expression of Alzheimer disease: the Nun Study," Journal of the American Medical Association, vol. 277, no. 10, pp. 813-817, 1997.

[9] H. Hénon, I. Durieu, D. Guerouaou, F. Lebert, F. Pasquier, and D. Leys, "Poststroke dementia: incidence and relationship to prestroke cognitive decline," Neurology, vol. 57, no. 7, pp. 1216-1222, 2001.

[10] J. C. De La Torre, "Is Alzheimer's disease a neurodegenerative or a vascular disorder? Data, dogma, and dialectics," Lancet Neurology, vol. 3, no. 3, pp. 184-190, 2004.

[11] "Cerebrovascular pathology in Alzheimer's disease," in Proceedings of a Conference, J. C. de la Torre and V. C. Hachinski, Eds., vol. 826, pp. 1-519, 1997.

[12] R. N. Kalaria and P. Ince, "Vascular factors in Alzheimer's disease," Annals of the New York Academy of Sciences, vol. 903, pp. 1-552, 2000.

[13] J. C. de la Torre, "Vascular pathophysiology in Alzheimer's disease," Neurobiology of Aging, vol. 21, pp. 153-383, 2000.

[14] J. C. De la Torre, R. Kalaria, K. Nakajima, and K. Nagata, "Alzheimer's Disease: vascular etiology and pathology: preface," Annals of the New York Academy of Sciences, vol. 977, pp. $1-526,2002$.

[15] L. K. Sztriha, D. Nemeth, T. Sefcsik, and L. Vecsei, "Carotid stenosis and the cognitive function," Journal of the Neurological Sciences, vol. 283, no. 1-2, pp. 36-40, 2009. 
[16] S. C. Johnston, E. S. O’Meara, T. A. Manolio et al., "Cognitive impairment and decline are associated with carotid artery disease in patients without clinically evident cerebrovascular disease," Annals of Internal Medicine, vol. 140, no. 4, pp. 237 I34, 2004.

[17] M. P. Spencer and J. M. Reid, "Quantitation of carotid stenosis with continuous-wave (C-W) Doppler ultrasound," Stroke, vol. 10, no. 3, pp. 326-330, 1979.

[18] A. V. Alexandrov, “The Spencer's curve: clinical implications of a classic hemodynamic model," Journal of Neuroimaging, vol. 17, no. 1, pp. 6-10, 2007.

[19] E. G. Lakatta, "Myocardial adaptations in advanced age," Basic Research in Cardiology, vol. 88, no. 2, pp. 125-133, 1993.

[20] K. L. Leenders, D. Perani, A. A. Lammertsma et al., "Cerebral blood flow, blood volume and oxygen utilization: normal values and effect of age," Brain, vol. 113, no. 1, pp. 27-47, 1990.

[21] L. Luo and F. I. M. Craik, "Aging and memory: a cognitive approach," Canadian Journal of Psychiatry, vol. 53, no. 6, pp. 346-353, 2008.

[22] P. W. Schofield, K. Marder, G. Dooneief, D. M. Jacobs, M. Sano, and Y. Stern, "Association of subjective memory complaints with subsequent cognitive decline in communitydwelling elderly individuals with baseline cognitive impairment," American Journal of Psychiatry, vol. 154, no. 5, pp. 609615, 1997.

[23] A. P. Haley, D. E. Forman, A. Poppas et al., "Carotid artery intima-media thickness and cognition in cardiovascular disease," International Journal of Cardiology, vol. 121, no. 2, pp. 148-154, 2007.

[24] A. S. Turk, I. Chaudry, V. M. Haughton et al., "Effect of carotid artery stenting on cognitive function in patients with carotid artery stenosis: preliminary results," American Journal of Neuroradiology, vol. 29, no. 2, pp. 265-268, 2008.

[25] R. Rao, "The role of carotid stenosis in vascular cognitive impairment," Journal of the Neurological Sciences, vol. 203-204, pp. 103-107, 2002.

[26] W. Hamster and H. C. Diener, "Neuropsychological changes associated with stenoses or occlusions of the carotid arteries. A comparative psychometric study," European Archives of Psychiatry and Neurological Sciences, vol. 234, no. 1, pp. 69-73, 1984.

[27] M. F. Folstein, S. E. Folstein, and P. R. McHugh, “"'Mini mental state". A practical method for grading the cognitive state of patients for the clinician," Journal of Psychiatric Research, vol. 12, no. 3, pp. 189-198, 1975.

[28] T. N. Tombaugh and N. J. McIntyre, "The Mini-Mental State Examination. a comprehensive review," Journal of the American Geriatrics Society, vol. 40, no. 9, pp. 922-935, 1992.

[29] Z. S. Nasreddine, N. A. Phillips, V. Bédirian et al., "The Montreal Cognitive Assessment, MoCA: a brief screening tool for mild cognitive impairment," Journal of the American Geriatrics Society, vol. 53, no. 4, pp. 695-699, 2005.

[30] I. Martinić-Popović, A. Lovrenčić-Huzjan, and V. Demarin, "Assessment of subtle cognitive impairment in stroke-free patients with carotid disease," Acta Clinica Croatica, vol. 48, no. 3, pp. 231-240, 2009.

[31] I. Martinic-Popovic, A. Lovrencic-Huzjan, A.-M. Simundic, A. Popovic, V. Seric, and V. Demarin, "Cognitive performance in asymptomatic patients with advanced carotid disease," Cognitive and Behavioral Neurology, vol. 24, no. 3, pp. 145151, 2011.

[32] V. Hachinski, C. Iadecola, R. C. Petersen et al., "National Institute of Neurological Disorders and Stroke-Canadian
Stroke Network vascular cognitive impairment harmonization standards," Stroke, vol. 37, no. 9, pp. 2220-2241, 2006.

[33] M. Silvestrini, I. Paolino, F. Vernieri et al., "Cerebral hemodynamics and cognitive performance in patients with asymptomatic carotid stenosis," Neurology, vol. 72, no. 12, pp. 10621068, 2009.

[34] E. R. Bossema, N. Brand, F. L. Moll, R. G. A. Ackerstaff, E. H. F. De Haan, and L. J. P. Van Doornen, "Cognitive functions in carotid artery disease before endarterectomy," Journal of Clinical and Experimental Neuropsychology, vol. 28, no. 3, pp. 357-369, 2006.

[35] P. Komulainen, M. Kivipelto, T. A. Lakka et al., "Carotid intima-media thickness and cognitive function in elderly women: a population-based study," Neuroepidemiology, vol. 28, no. 4, pp. 207-213, 2007.

[36] J. R. Romero, A. Beiser, S. Seshadri et al., "Carotid artery atherosclerosis, MRI indices of brain ischemia, aging, and cognitive impairment: the framingham study," Stroke, vol. 40, no. 5, pp. 1590-1596, 2009.

[37] J. H. Fergenbaum, S. Bruce, J. D. Spence et al., "Carotid atherosclerosis and a reduced likelihood for lowered cognitive Performance in a Canadian first nations population," Neuroepidemiology, vol. 33, no. 4, pp. 321-328, 2009. 


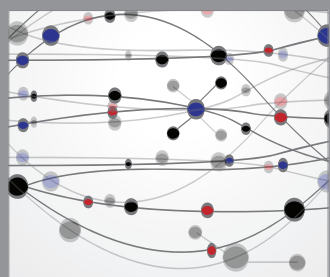

The Scientific World Journal
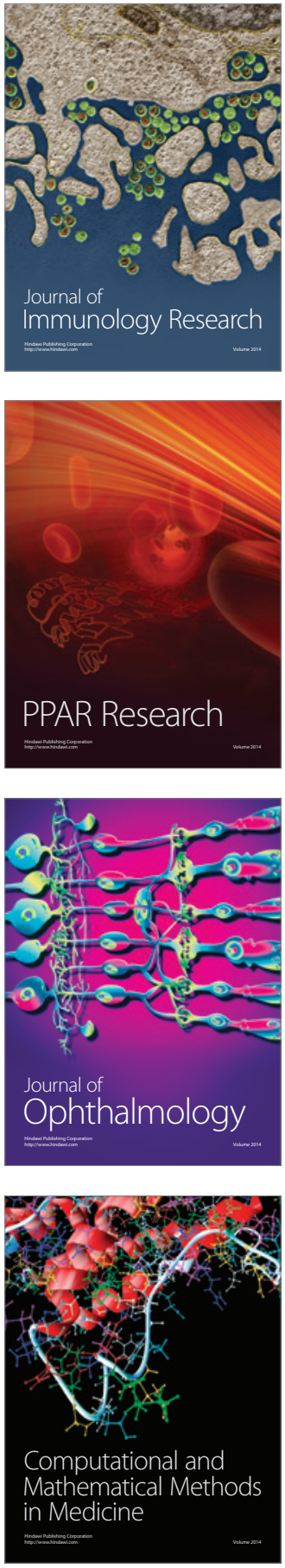

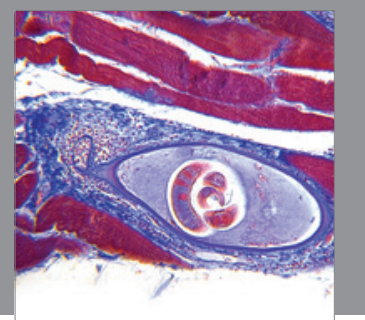

Gastroenterology

Research and Practice
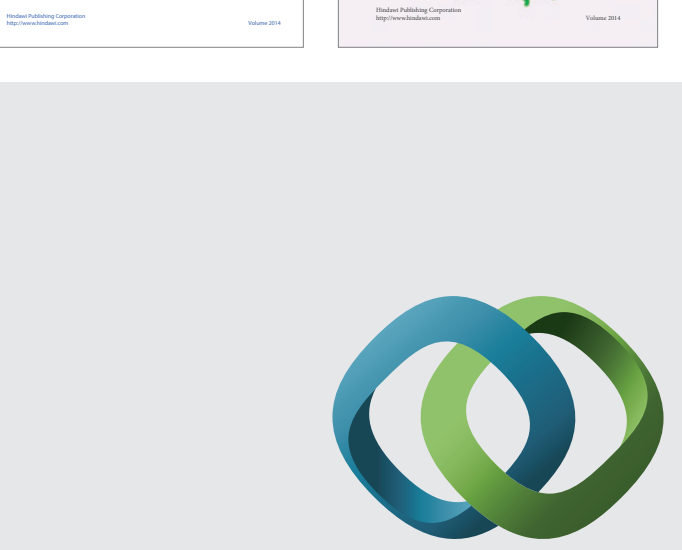

\section{Hindawi}

Submit your manuscripts at

http://www.hindawi.com
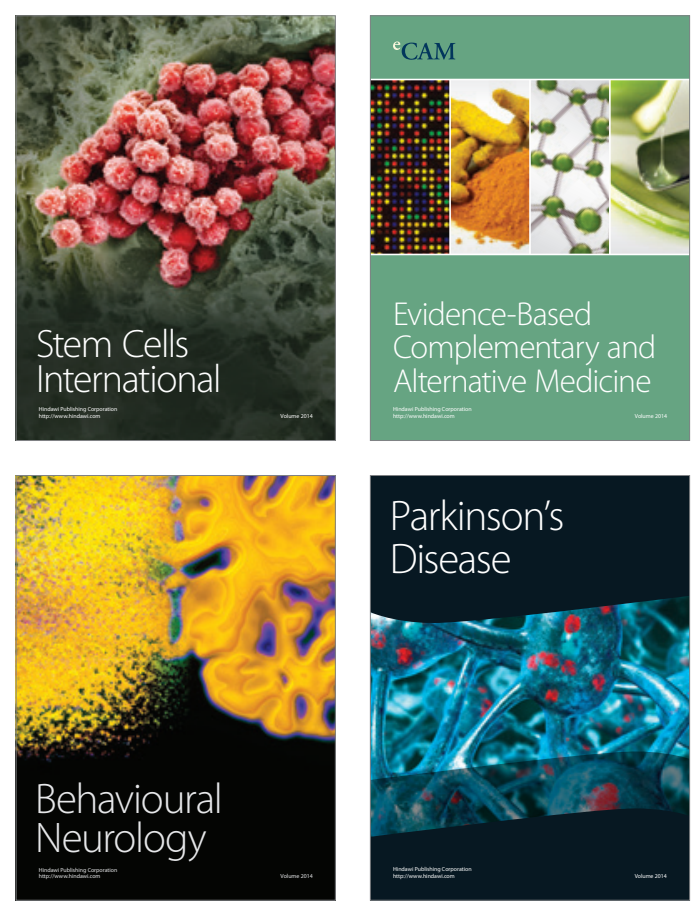

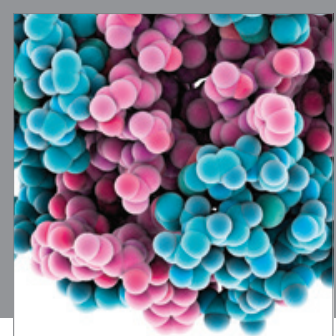

Journal of
Diabetes Research

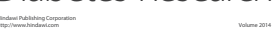

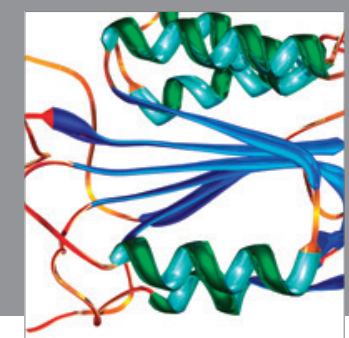

Disease Markers
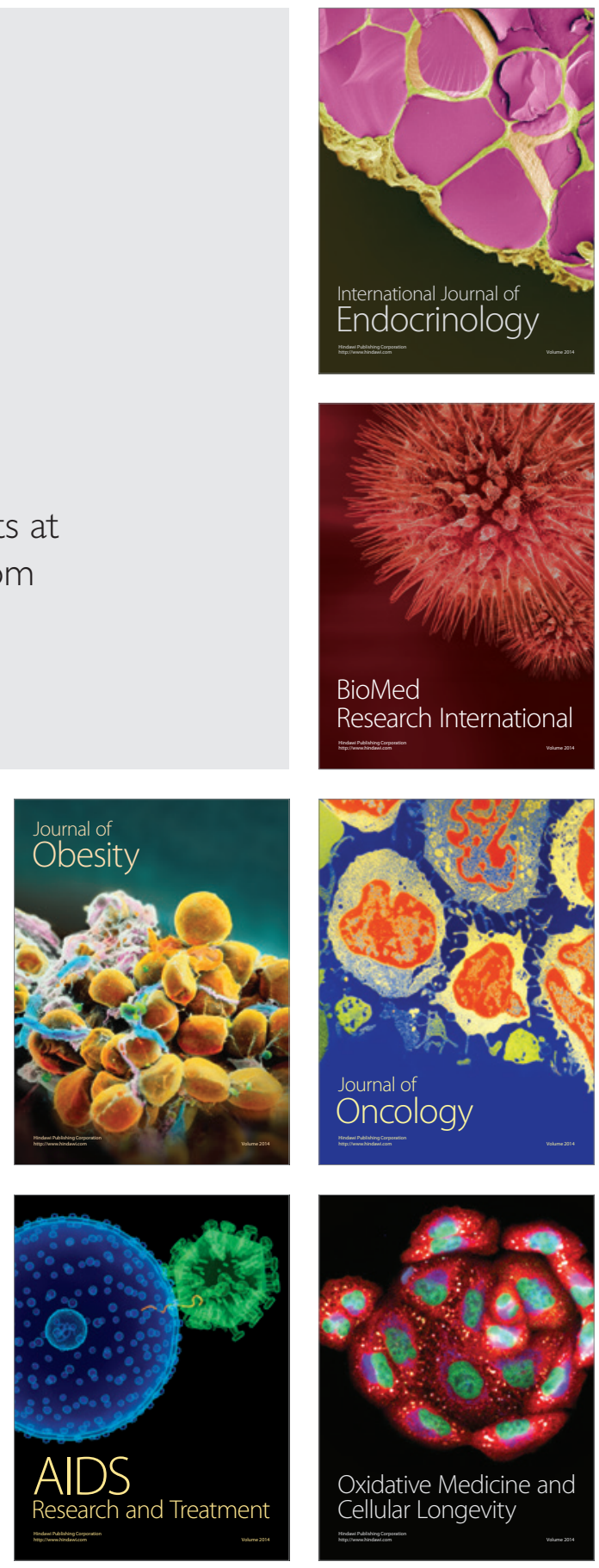